\title{
Immunological Evidence for the Role of Mycobacteria in Sarcoidosis: A Meta-Analysis
}

\author{
Chuling Fang, Hui Huang, Zuojun $\mathrm{Xu}^{*}$ \\ Department of Respiratory Medicine, Peking Union Medical College Hospital, Chinese Academy of Medical \\ Sciences \& Peking Union Medical College, Beijing, China \\ *xuzj@ hotmail.com
}

\section{Abstract}

\section{Background}

Sarcoidosis is a granulomatous disease, the etiology of which is currently unknown. The role of mycobacteria in the etiology of sarcoidosis has been extensively investigated. In this meta-analysis, we assessed the immunological evidence of the possible role of mycobacteria in the pathogenesis and development of sarcoidosis.

Immunological Evidence for the Role of Mycobacteria in Sarcoidosis: A Meta-Analysis. PLoS ONE 11(8): e0154716. doi:10.1371/journal.pone.0154716

Editor: Gernot Zissel, Universitatsklinikum Freiburg, GERMANY

Received: January 13, 2016

Accepted: April 18, 2016

Published: August 1, 2016

Copyright: @ 2016 Fang et al. This is an open access article distributed under the terms of the Creative Commons Attribution License, which permits unrestricted use, distribution, and reproduction in any medium, provided the original author and source are credited.

Data Availability Statement: All relevant data are within the paper.

Funding: This work was supported by the National Natural Science Foundation of China (No. 81170055); and Key Projects in the National Science \& Technology Pillar Program during the Twelfth Fiveyear Plan Period (2012BAI05B02), URL: http://www. letpub.com.cn/?page=grant\&name=\&person=\%E5\% $\mathrm{BE} \% 90 \% \mathrm{E} 4 \% \mathrm{BD} \% 9 \mathrm{C} \% \mathrm{E} 5 \% 86 \% 9 \mathrm{~B} \& \mathrm{no}=$

\&company=\&addcomment_s1=662\&addcomment_ s2 $=0 \&$ addcomment_s3 $=0 \&$ money $1=\&$ money $2=$ \&startTime=2011\&endTime=2015\&subcategory= \&searchsubmit=true\&submit.x $=59$ \&submit. $y=$ 13\#fundlisttable. The funders had no role in study

\section{Methods}

We performed a systematic search of relevant articles from PubMed, Embase and Cochrane Library databases published between January 1990 and October 2015. Data extracted from the articles were analyzed with Review Manager 5.3 (Cochrane Collaboration, Oxford, UK).

\section{Results}

In this meta-analysis, 13 case-control studies (733 participants) were considered eligible according to our criteria. Methodological quality was assessed using the Newcastle-Ottawa Scale (NOS). The positivity incidence of the immune response (either the cell-mediated response or humoral response) in sarcoidosis patients was significantly higher than that in controls, as determined using fixed-effects model. The odds ratio (OR) of the positivity incidence of T-cell response in the patients with sarcoidosis versus the controls with PPD- or unknown PPD status was 5.54 (95\% Cl 3.56-8.61); the ORs were 16.70 (95\% Cl 8.19$34.08)$ and 1.48 (95\% $\mathrm{Cl} 0.74-2.96)$ for the two subgroups with PPD- controls and unknown PPD status respectively. However, the OR of the positivity incidence in patients with sarcoidosis versus PPD+ controls (latent tuberculosis infection; LTBI) was 0.26 (95\% 0.100.66). Regarding the humoral response, pooled analysis of the positivity incidence revealed an OR $(95 \% \mathrm{Cl})$ of 20.43 (5.53-75.53) for the patients with sarcoidosis versus controls; the ORs were 11.93 (95\% Cl 2.15-66.27) and 41.97 (95\% Cl 5.24-336.15) in two subgroups of controls with PPD- and unknown PPD statuses respectively. Data on heterogeneity and evidence of publication bias were examined. 
design, data collection and analysis, decision to publish, or preparation of the manuscript.

Competing Interests: The authors have declared that no competing interests exist.

\section{Conclusions}

This meta-analysis confirmed the existence of an association between mycobacteria (especially M.tuberculosis) and sarcoidosis. The current available evidence indicates that some insoluble mycobacterial antigens that preferentially within the body are involved in the pathogenesis of sarcoidosis rather than the whole mycobacteria and that they elicit a type IV immune response.

\section{Introduction}

Mycobacterium tuberculosis (M. tuberculosis) is the second most common infectious cause of death in adults worldwide (HIV is the most common). The human host serves as a natural reservoir for M. tuberculosis, which is an intracellular obligate and aerobic bacillus that multiplies within macrophages. The bacterium triggers the production of free radicals and avoids being killed by the same radicals [1]. In the lungs, macrophages produce cytokines and chemokines that attract other phagocytic cells, including monocytes, other alveolar macrophages, and neutrophils, which eventually form a nodular granulomatous structure called a tubercle. The ability of $M$. tuberculosis to efficiently establish latent infection has enabled it to spread to nearly one-third of individuals worldwide.

Sarcoidosis is a systemic granulomatous disease characterized by the formation of epithelioid cell granulomas (and is accompanied by an infiltration of inflammatory cells) without caseous necrosis. Multisystem granulomatous inflammation involving the lungs, lymph nodes, skin, eyes, heart, and muscles is a hallmark of sarcoidosis. Infectious and genetic factors, as well as autoimmunity, are considered to be potential causes of SA [2-4]. In addition, mycobacterial antigens, such as the 6-kDa ESAT6, catalase-peroxidase (mKatG), superoxide dismutase A (Sod A) and M. tuberculosis heat shock proteins (Mtb-hsp) have been suggested to be infectious factors for the pathogenesis of SA [5].

In the middle of the 20th century, M. tuberculosis was isolated from several sarcoidosis patients by Scadding [6]. The relationship between $M$. tuberculosis and sarcoidosis has since been explored with increasing frequency. The advent of molecular biology invigorated the search for mycobacterial DNA in sarcoidosis patients $[7,8]$. One meta-analysis conducted by Gupta [9] compiled 31 published studies on the presence of mycobacteria in sarcoidosis patients. The authors concluded that the evidence from pooled analysis favored the existence of an association between mycobacteria and sarcoidosis.

Recent studies evaluating immunological evidence of mycobacterial antigens in sarcoidosis patients has renewed interest in the role of mycobacteria in sarcoidosis [10], indicating that $M$. tuberculosis antigens could be involved in the pathogenesis of sarcoidosis. Many studies on the T-cell response to $M$. tuberculosis antigens have been conducted using an interferon gamma release assay (IGRA), which has been proven to be more efficient than the TST (tuberculin skin test) [11]. We conducted a meta-analysis of the available published literature to analyze the role of mycobacteria in the pathogenesis of sarcoidosis.

\section{Materials and Methods}

\section{Search strategy and selection criteria}

The aim of this meta-analysis was to collect all publicly available studies on the immune response to $M$. tuberculosis antigens in patients with sarcoidosis. To identify relevant articles 
for inclusion in this review, all authors independently searched PubMed, Embase, and Cochrane Library databases for relevant studies published from 1990-2015 using the following terms: sarcoidosis AND mycobacteria (OR) mycobacterium; sarcoidosis AND mycobacterium tuberculosis; and sarcoidosis AND tuberculosis. Additional eligible studies were identified by reviewing the references of all retrieved- literatures and review articles addressing the relationship between sarcoidosis and mycobacteria. The outcome measure of all included studies was positivity incidence of the immune response to $M$. tuberculosis antigens. No language or geographic restrictions were imposed on identified studies.

\section{Data abstraction and Quality Assessment}

Paired reviewers (Chuling Fang and Hui Huang) independently evaluated studies for eligibility using a two-stage procedure. During the first stage, all identified abstracts were evaluated to ensure that they were involved in the relationship between sarcoidosis and mycobacteria. All potentially relevant studies were retrieved and selected for the second stage of the procedure, in which a full-text review was performed to determine whether the studies provided the positivity incidence of the immune response. Any disagreements were resolved by discussion and consensus. Data concerning the immune response to M. tuberculosis antigens were extracted onto standardized data collection forms by the two reviewers, and the verified data were entered into a Microsoft Excel spreadsheet (XP professional edition; Microsoft Corp, Redmond, WA, USA). The following items were extracted: 1) the publication details including the study geography (country or region), year of publication, first author and other citation details; 2) the number of patients with sarcoidosis and the type of mycobacterium (M. tuberculosis or other mycobacterium) used in analysis; 3 ) details of the technique used to evaluate the immune response in samples; 4) the health condition of the control subjects and whether details on TST outcomes were provided and 5) the numbers of the samples with an active immune response to mycobacterial antigens for patients with sarcoidosis and control. No attempt was made to include unpublished data. Methodological quality was assessed by the Newcastle-Ottawa Scale (NOS).

\section{Data Synthesis and Analysis}

The included articles were analyzed using Cochrane Collaboration Review Manager statistical software (version 5.3; Cochrane Collaboration, Oxford, UK). To calculate the percentage of sarcoidosis samples with a positive immune reaction to M. tuberculosis, binomial proportions were used in which the numerator was sarcoid samples with active immune response to $M$. tuberculosis antigens and the denominator was the total number of study samples. As dichotomous outcomes, the percentage of sarcoid samples with a positive immune response out of the total samples versus the same percentage of control samples was expressed as an odds ratio (OR) and a 95\% confidence interval (CI). The fixed-effects model was used, and the randomeffects model weighted by the Mantel-Haenszel method was utilized for data with significant heterogeneity (P value of $\mathrm{x}^{2}$ test $<0.05$ and $I^{2}>50 \%$ ). $\mathrm{P}<0.05$ was considered statistically significant.

\section{Assessment of heterogeneity}

The impact of heterogeneity on the pooled estimates of individual outcomes of meta-analysis was assessed using the Chi-squared test and/or $\mathrm{I}^{2}$ test (this test measures the extent of inconsistency among studies' results and is interpreted as the approximate proportion of total variation in study estimates due to heterogeneity rather than sampling error). An $\mathrm{I}^{2}$ value $>50 \%$ indicates significant heterogeneity. 


\section{Assessment of publication bias}

A funnel plot test was used to assess publication bias. The funnel plot is a measurement of the log of the OR (on the X-axis; a measurement of diagnostic accuracy) against the standard error of the log of the OR (on the Y-axis; an indicator of sample size). Each point represents a study in meta-analysis.

\section{Results \\ Study Identification}

The process of identifying eligible studies is summarized in Fig 1. Studies were retrieved using the aforementioned process. After an evaluation of the title and abstract, 71 studies were subjected to further assessments. After full-text reviews, 13 trials (13articles) were included in meta-analysis, and related data were extracted. Detailed characteristics of the included studies are provided in Table 1. The 13 studies [12-24] published between 1996 and 2014 enrolled a total of 733 participants: 555 involving the T-cell response (253 in the sarcoidosis group, 302 in the control group with PPD-, unknown PPD status or PPD+ (LTBI)); and 178 Involving the humoral response (100 in the sarcoidosis group and 78 in the control group). Six of the trials were conducted in the USA [13, 16-19, 22], two were performed in India [14, 20], and one each was carried out in Germany [15], the Netherlands [12], Poland [21], Sweden [23] and Australia [24] respectively.

All studies performed detailed immunology analysis and the meta-analysis included studies conducted around the world (Table 1). In total, the studies included 353 samples (235 blood samples and 118 BALF samples) from 353 patients with proven sarcoidosis as well as 380 samples (224 blood samples and 156 BALF samples) from 380 controls (with another lung disease or healthy with PPD-, PPD+ or unknown PPD status). Nine studies [12-19, 24] on T-cell immune response were included. Among them, the control group in two studies [13, 16] were composed of PPD- individuals, those in other three studies $[12,14,15)]$ contained individuals with an unknown PPD status, and the remaining four [17-19,24] used two control groups at the same time, consisted of PPD+ (LTBI) individuals and PPD- individuals respectively. Four studies [20-23] were included with humoral immune responses, the control groups of two studies [21,22] among them were PPD-, and the other two studies [20,23] were with unknown PPD subjects.

The quality scores of all 13 included studies were above five points, ranging from five to nine points. Five studies $[12,13,16,20,24]$ achieved the highest score, and four others [14, 15, 18,19 ] also achieved high score (Table 2). All studies were case control studies. All of the cases had adequate definitions with independent validation. Four studies [12, 13, 16, 20] controlled age and sex to insure the comparability of cases and controls. In addition, four studies [20-23] utilized three different methods (ELISA, immunohistochemical analysis, or protein immunoblotting) to test the humoral response to M.tuberculosis antigens. Further, nine studies [12-19, 24] used IGRA to test the T-cell response to these antigens.

\section{T-cell immune response}

Nine trials $[12-19,24]$ reported the positivity incidence of the T-cell immune response to $M$. tuberculosis specific antigens. Among the 253 samples in the sarcoidosis group, 126 exhibited a positive immune response to $M$. tuberculosis specific antigens, as demonstrated by immunology. The combined results of the nine trials revealed that the sarcoidosis patients had a significantly higher positivity rate of the T-cell immune response to $M$. tuberculosis specific antigens compared to the controls with PPD- or unknown PPD status (OR = 5.54, 95\% CI: 3.56-8.61, 

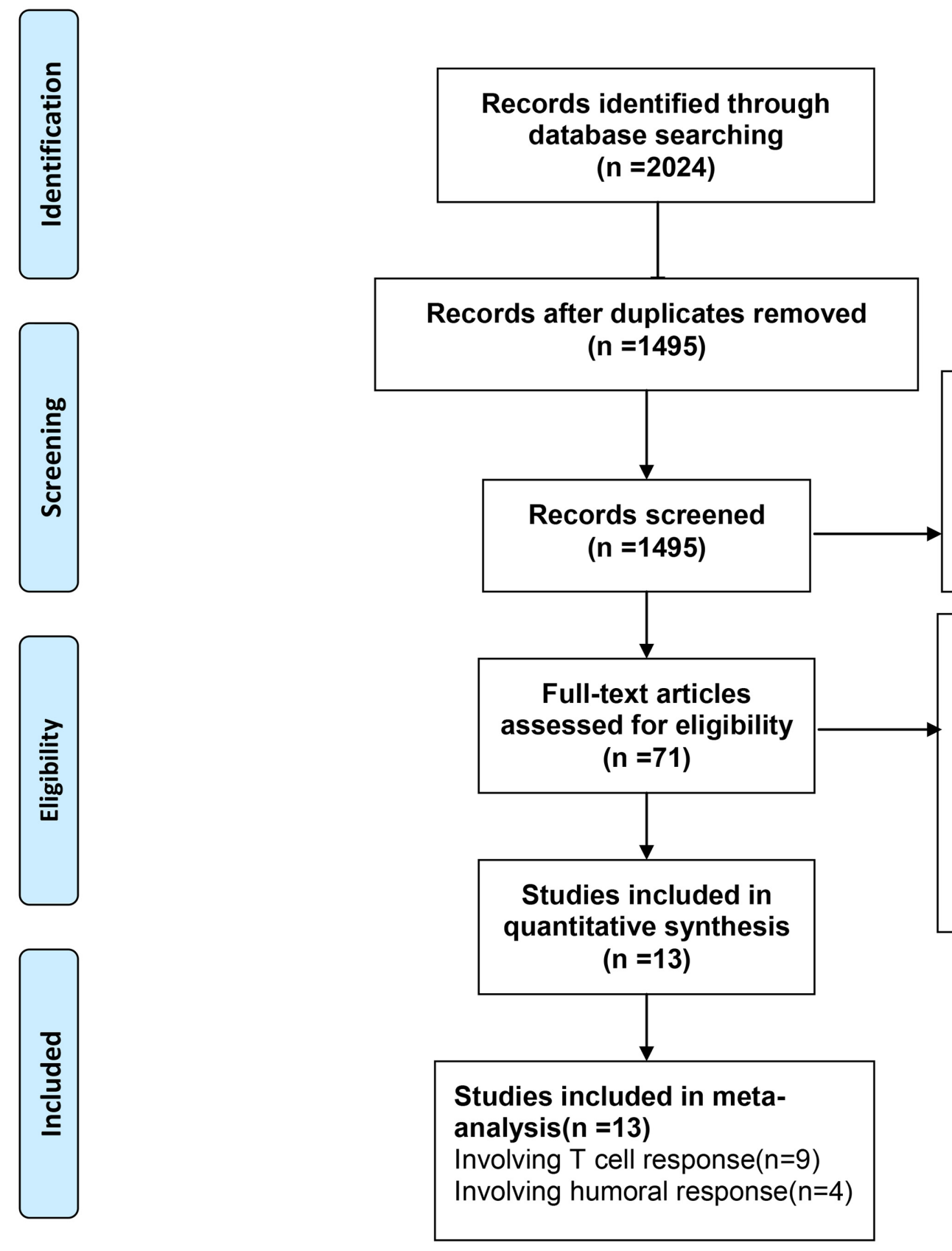

Records excluded $(\mathrm{n}=$ 1424) :

not involve

sarcoidosis $(n=1319)$

review $(n=29)$

case report $(n=76)$

\section{Full-text articles excluded,} with reasons $(n=58)$

studies with molecular evidence for the role of mycobacterium in sacoidosis $(n=36)$ studies not providing immune response positivity $(n=20)$ studies not providing controls $(n=2)$

Fig 1. Flow diagram of assessment of studies identified in meta-analysis.

doi:10.1371/journal.pone.0154716.g001

$\mathrm{P}<0.00001$ ) (Fig 2). Combined analysis of six trials $[13,16-19,24]$ revealed that the sarcoidosis patients had a significantly higher positivity rate than the subgroup of PPD-controls $(\mathrm{OR}=16.70,95 \% \mathrm{CI}: 8.19-34.08, \mathrm{P}<0.00001)$. Further, combined analysis of the three trials $[12,14,15]$ revealed that the positivity rate of immune response in the sarcoidosis group did not significantly differ between the sarcoidosis patients and subgroup of controls with an unknown PPD status ( $\mathrm{OR}=1.48,95 \% \mathrm{CI}$ : 0.74-2.96, $\mathrm{P}=0.26$ ) (Fig 2). An additional combined analysis of four trials $[17-19,24]$ revealed that the sarcoidosis patients had significantly lower positivity rate than the PPD+ controls (LTBI) $(\mathrm{OR}=0.26,95 \% \mathrm{CI}: 0.10-0.66, \mathrm{P}=0.004)$ (Fig 3). 
Table 1. Studies evaluating the role of mycobacteria in sarcoidosis using immunology analysis.

\begin{tabular}{|c|c|c|c|c|c|c|c|c|c|c|}
\hline \multirow[t]{2}{*}{ First author } & \multirow[t]{2}{*}{ Year } & \multirow[t]{2}{*}{ Site } & \multirow[t]{2}{*}{ Technique } & \multicolumn{4}{|c|}{ Patients } & \multicolumn{3}{|c|}{ Control } \\
\hline & & & & $\mathbf{n}$ & tissue/fluid & $\mathbf{N}$ & mycobacteria & $\mathbf{n}$ & diagnosis & $\mathbf{N}$ \\
\hline \multicolumn{11}{|c|}{ T-cell immune responses } \\
\hline Hofland & 2014 & Netherlands & $\begin{array}{l}\text { IFN-y ELISpot measuring } \\
\text { responses to PPD }\end{array}$ & 5 & BALF & 32 & MTB & 13 & $\begin{array}{l}\text { Other causes of interstitial lung } \\
\text { disease, unknown PPD }\end{array}$ & 86 \\
\hline Ahmadzai & 2012 & Australia & $\begin{array}{l}\text { IFN-y ELISpot measuring } \\
\text { responses to ESAT- } 6 \text { and } \\
\text { KatG }\end{array}$ & 10 & $\begin{array}{l}\text { Peripheral blood } \\
\text { mononuclear cells } \\
\text { (PBMCs) }\end{array}$ & 16 & MTB & $\begin{array}{l}4 / \\
5\end{array}$ & Healthy, PPD-/ PPD+(LTBI) & $\begin{array}{c}17 / \\
5\end{array}$ \\
\hline $\begin{array}{l}\text { Oswald- } \\
\text { Richter }\end{array}$ & 2012 & USA & $\begin{array}{l}\text { Flow cytometry measuring } \\
\text { IFN-y production in } \\
\text { response to ESAT- } 6\end{array}$ & 17 & BALF & 27 & MTB & 2 & Disease control, PPD- & 14 \\
\hline Gupta & 2011 & India & $\begin{array}{l}\text { QFT-GIT measuring } \\
\text { responses to ESAT-6, } \\
\text { CFP-10 and TB 7.7 }\end{array}$ & 13 & PBMCs & 38 & MTB & 5 & Healthy, PPD<10mm & 18 \\
\hline Horster & 2009 & Germany & $\begin{array}{l}\text { IFN-y ELISPOT measuring } \\
\text { responses to ESAT- } 6\end{array}$ & 7 & BALF & 15 & MTB & 7 & $\begin{array}{l}\text { Bacterial and viral pneumonia, } \\
\text { cryptogenic organizing } \\
\text { pneumonia, and bronchogenic } \\
\text { carcinoma, } P P D \leq 15 \mathrm{~mm}\end{array}$ & 29 \\
\hline $\begin{array}{l}\text { Oswald- } \\
\text { Richter }\end{array}$ & 2009 & USA & $\begin{array}{l}\text { Flow cytometry measuring } \\
\text { IFN-yproduction in } \\
\text { responses to ESAT- } 6 \text { and } \\
\text { KatG }\end{array}$ & 32 & BALF & 44 & MTB & 1 & Disease control, PPD- & 27 \\
\hline Drake & 2007 & USA & $\begin{array}{l}\text { Flow cytometry measuring } \\
\text { IFN-yproduction in } \\
\text { responses to ESAT- } 6 \text { and } \\
\text { KatG }\end{array}$ & 15 & PBMCs & 26 & MTB & $\begin{array}{l}1 / \\
7\end{array}$ & Healthy, PPD-/ PPD+ (LTBI) & $\begin{array}{c}24 / \\
8\end{array}$ \\
\hline Carlisle & 2007 & USA & $\begin{array}{l}\text { ELISPOT assessing Th1 } \\
\text { responses to sodA, mkatG } \\
\text { and ESAT-6 }\end{array}$ & 12 & PBMCs & 30 & MTB & $\begin{array}{l}1 / \\
6\end{array}$ & Healthy, PPD-/ PPD+ (LTBI) & $\begin{array}{l}26 / \\
10\end{array}$ \\
\hline Hajizadeh & 2007 & USA & $\begin{array}{l}\text { IFN-y ELISPOT measuring } \\
\text { responses to Antigen } 85 \mathrm{~A}\end{array}$ & 15 & PBMCs & 25 & MTB & $\begin{array}{l}2 / \\
14\end{array}$ & Healthy, PPD-/ PPD+ (LTBI) & $\begin{array}{l}22 / \\
16\end{array}$ \\
\hline \multicolumn{11}{|c|}{ humoral immunity } \\
\hline Agarwal & 2012 & India & $\begin{array}{l}\text { Humoral responses to } \\
\text { ESAT-6 and CFP-10 }\end{array}$ & 11 & blood samples & 18 & MTB & 0 & healthy, unknown PPD & 20 \\
\hline Dubaniewicz & 2006 & Poland & $\begin{array}{l}\text { Anti-Mtb-hsp70, -Mtb- } \\
\text { hsp65 and -Mtb-hsp16 } \\
\text { antibodies }\end{array}$ & 12 & blood samples & 37 & MTB & 0 & PPD- & 18 \\
\hline Song & 2005 & USA & IgG antibodies to mKatG & 12 & PBMCs & 25 & MTB & 0 & healthy PPD- & 11 \\
\hline El-Zaatari & 1996 & Sweden & $\begin{array}{l}\text { Humoral reactivities to } M \text {. } \\
\text { paratuberculosis } \\
\text { recombinant clones } \\
\text { expressing p36 }\end{array}$ & 7 & blood samples & 7 & MPTB & 13 & $\begin{array}{c}\text { Ulcerative colitis, } \\
\text { noninflammatory bowel disease } \\
\text { or healthy, unknown PPD }\end{array}$ & 38 \\
\hline
\end{tabular}

BALF $=$ Bronchoalveolar lavage fluid, PBMCs $=$ Peripheral blood mononuclear cells, LTBI $=$ Latent tuberculosis infection.

doi:10.1371/journal.pone.0154716.t001

Heterogeneity analysis revealed that there was no substantial heterogeneity in either the PPDor unknown PPD control subgroups as assessed by using the $\mathrm{I}^{2}$ statistics (subgroup of PPDcontrols $(\mathrm{Q}$ (d.f. $=5)=4.57, \mathrm{P}=0.47, \mathrm{I}^{2}=0 \%$ ); subgroup of controls with unknown PPD status $\left(\mathrm{Q}(\mathrm{d} . \mathrm{f} .=2)=1.24, \mathrm{P}=0.54, \mathrm{I}^{2}=0 \%\right)$ (Fig 2$)$. However, the heterogeneity between these two subgroups was substantial ( $\mathrm{Q}($ d.f. $\left.=1)=22.84, \mathrm{P}<0.00001, \mathrm{I}^{2}=95.6 \%\right)$ (Fig 2). Further, no substantial heterogeneity was detected in the $\mathrm{PPD}+$ control group $(\mathrm{Q}(\mathrm{d} . \mathrm{f} .=3)=0.76 \mathrm{P}=0.86$, $\mathrm{I}^{2}=0 \%$ ) (Fig 3). Publication bias was not evident, as estimated by generating a funnel plot for the studies on the T-cell immune response to M. tuberculosis specific antigens (Fig 4a and 4b). 


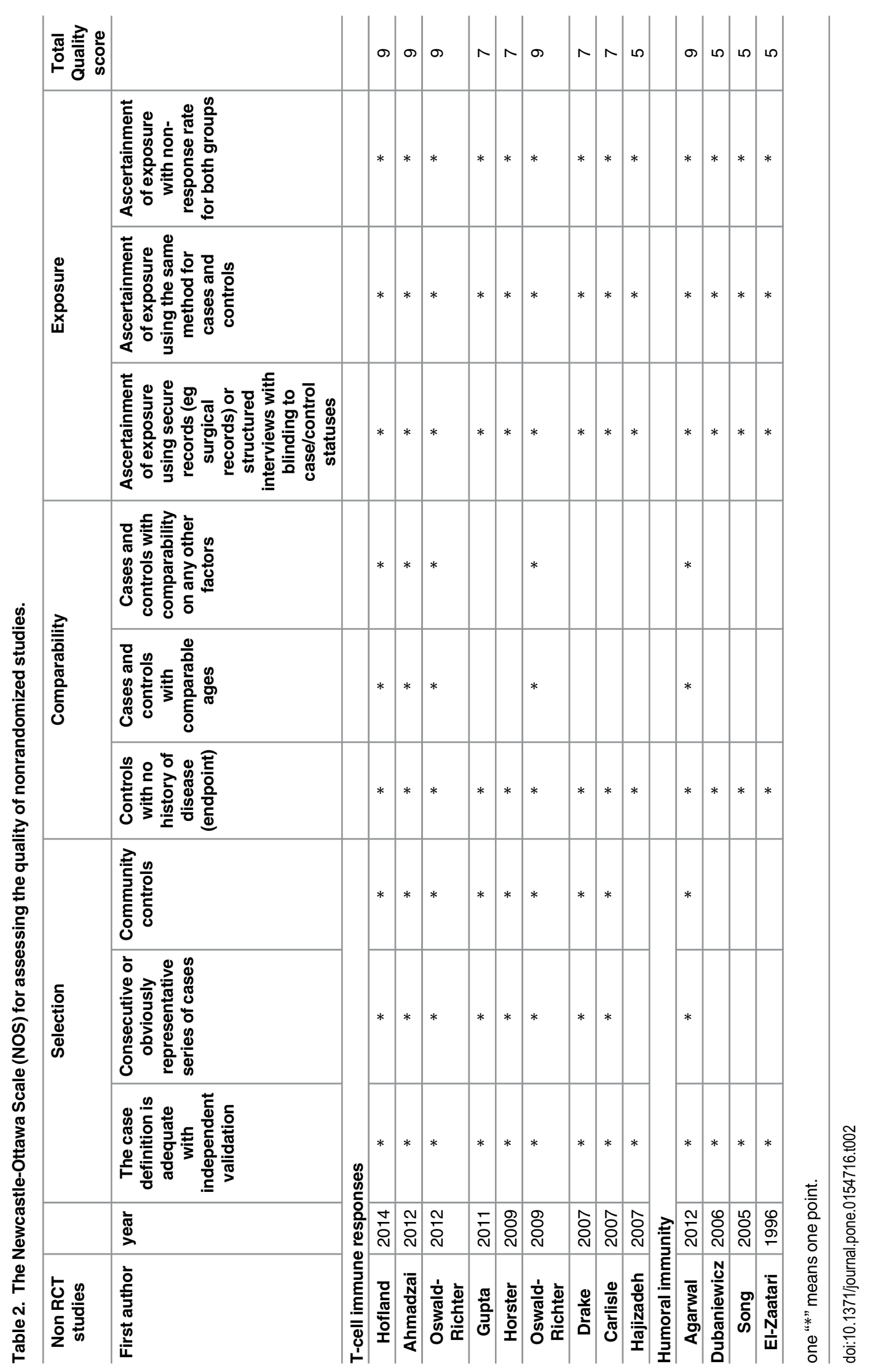




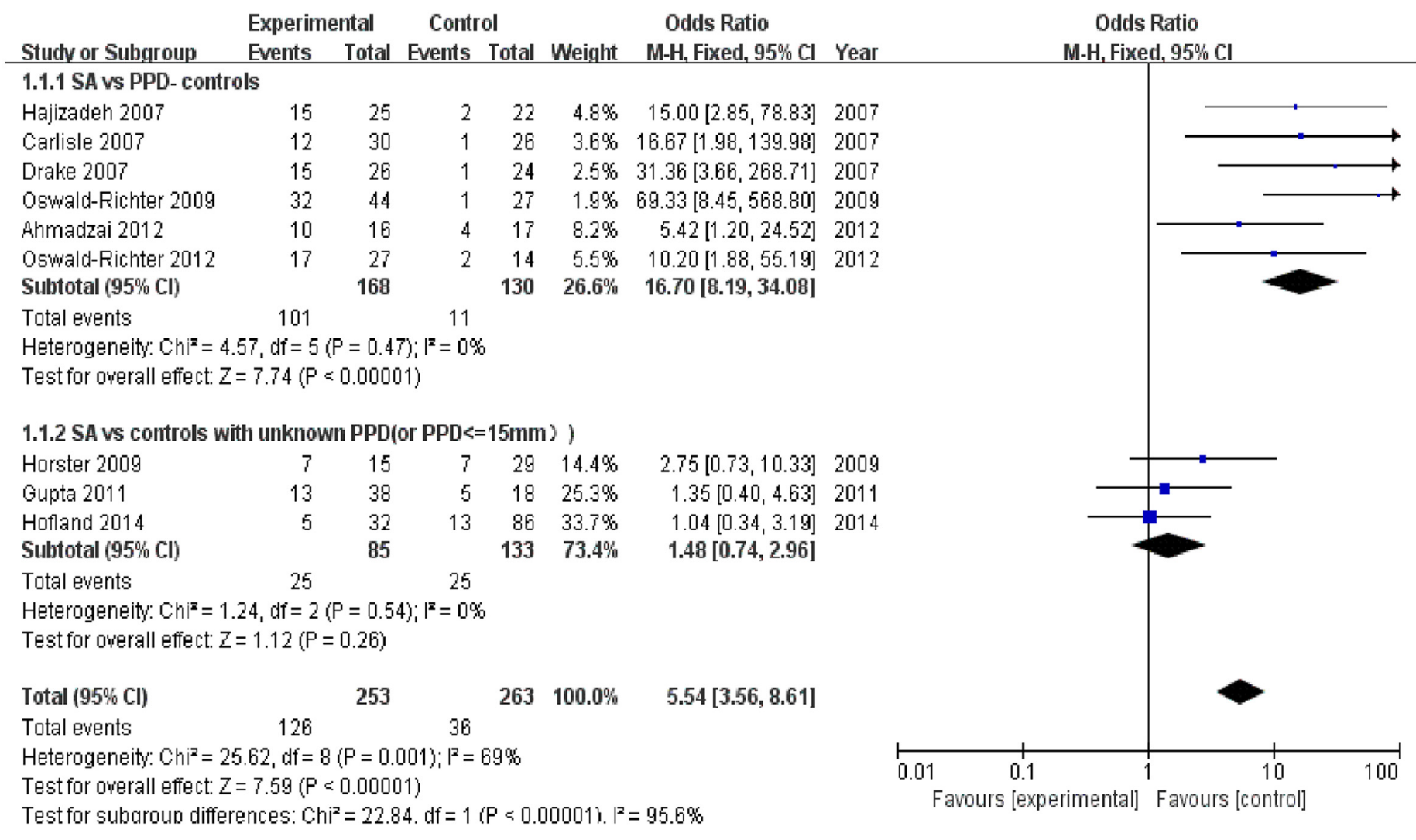

Fig 2. Forest plot of trials analyzing the positivity incidence of the T-cell response to $M$. tuberculosis antigens in sarcoidosis patients versus controls with PPD- and unknown PPD statuses.

doi:10.1371/journal.pone.0154716.g002

\section{Humoral response}

Four trials [20-23] reported the positivity incidence of the humoral immune response to $M$. tuberculosis specific antigens. Of the100 samples in the sarcoidosis group, 55 exhibited a

Experimental Control Odds Ratio Odds Ratio

Studv or Subgroup Events Total Events Total Weight M-H, Fixed, $95 \% \mathrm{Cl}$

\begin{tabular}{|c|c|c|c|c|c|c|c|c|}
\hline Anmadzai 2012 & 10 & 16 & 5 & 5 & $15.6 \%$ & $0.15[0.01,3.12]$ & $\longmapsto$ & - \\
\hline Carlisie 2007 & 12 & 30 & 6 & 10 & $27.2 \%$ & $0.44[0.10,1.92]$ & & - \\
\hline Drake 2007 & 15 & 26 & 7 & 8 & $22.8 \%$ & $0.19[0.02,1.82]$ & & \\
\hline Hajizadeh 2007 & 15 & 25 & 14 & 16 & $34.4 \%$ & $0.21[0.04,1.15]$ & & \\
\hline Total (95\% Cl) & & 97 & & 39 & $100.0 \%$ & $0.26[0.10,0.66]$ & & \\
\hline Total events & 52 & & 32 & & & & & \\
\hline \multicolumn{7}{|c|}{ Heterogeneity chi ${ }^{2}=0.76, d f=3(P=0.86) ; 1^{2}=0 \%$} & 0.01 & 10 \\
\hline
\end{tabular}

Fig 3. Forest plot of trials analyzing the positivity incidence of T-cell response to $M$. tuberculosis antigens in sarcoidosis patients versus PPD + controls (LTBI). LTBI = latent tuberculosis infection.

doi:10.1371/journal.pone.0154716.g003 

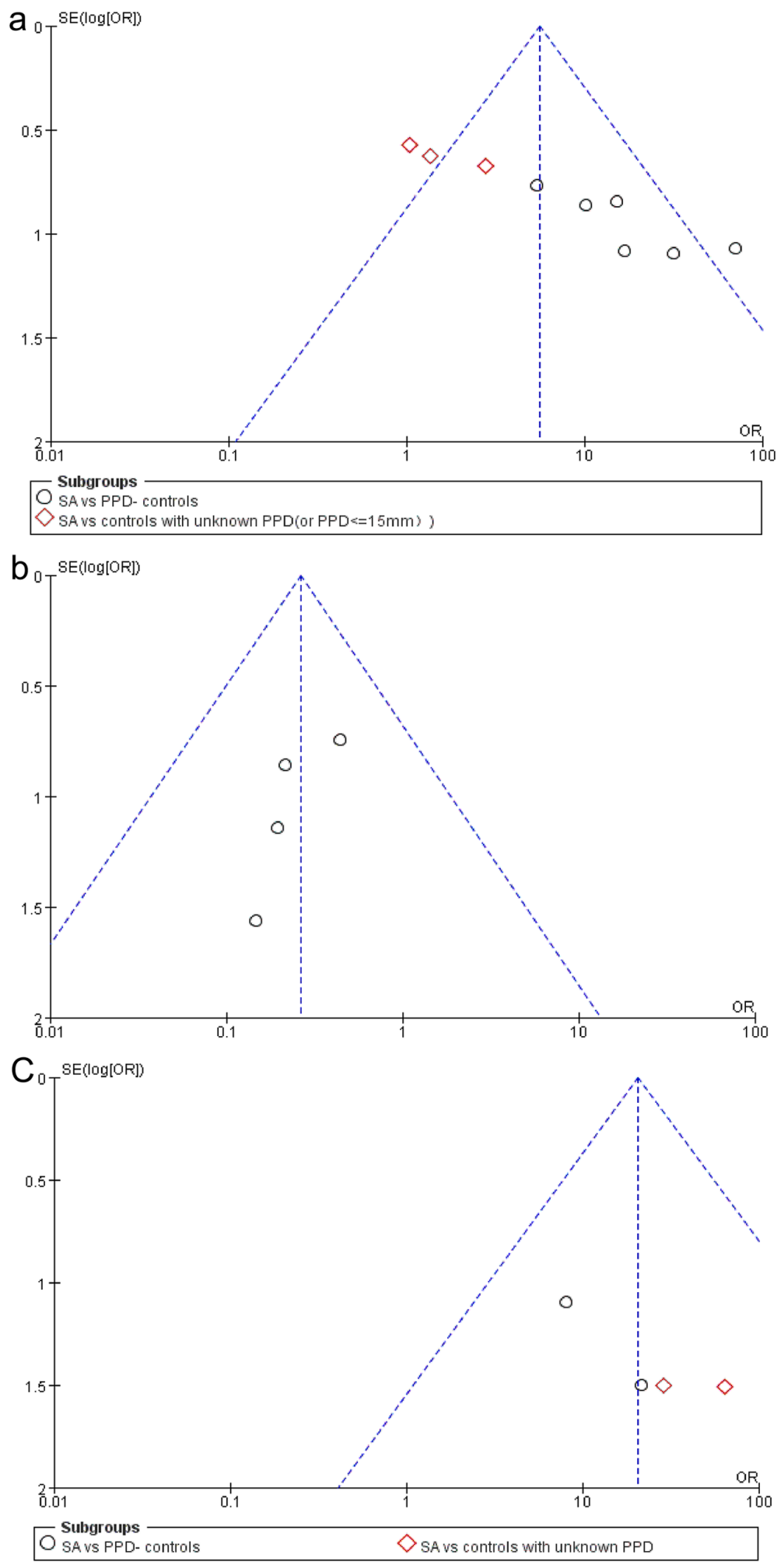

Fig 4. Funnel plot assessing publication bias. a. Funnel plot for the studies on the T-cell response to M. tuberculosis antigens with controls of PPD- or unknown PPD status; $b$. Funnel plot for the studies on the T-cell response to $M$. tuberculosis antigens with PPD+controls; $c$. Funnel plot for studies on the humoral response to $M$. tuberculosis antigens. SA = sarcoidosis.

doi:10.1371/journal.pone.0154716.g004 


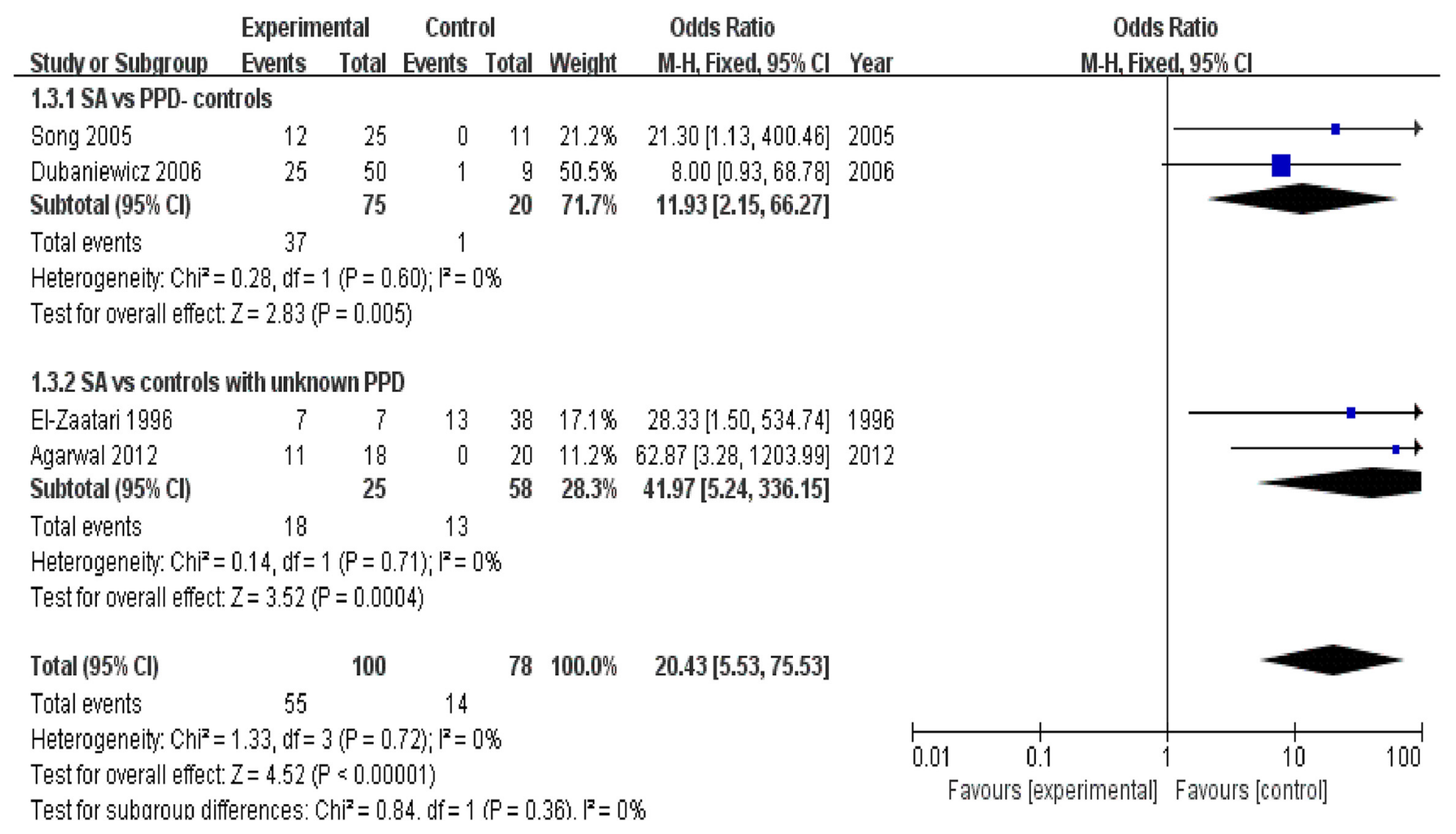

Fig 5. Forest plot of trials analyzing the positivity incidence of the humoral response to $M$. tuberculosis antigens in sarcoidosis patients versus controls.

doi:10.1371/journal.pone.0154716.g005

positive immune response to M. tuberculosis specific antigens, as demonstrated by ELISA and immunohistochemical analysis. The combined results of four trials [20-23] revealed that the sarcoidosis group had a significantly higher positivity rate of humoral response to $M$. tuberculosis specific antigens than the control group $(\mathrm{OR}=20.43,95 \% \mathrm{CI}$ : 5.53-75.53, $\mathrm{P}<0.0001)$. Further, the combined results of two trials $[21,22]$ revealed that the sarcoidosis group had a significantly higher positivity rate of the humoral immune response to $M$. tuberculosis specific antigens than subgroup of PPD- controls ( $\mathrm{OR}=11.93,95 \% \mathrm{CI}: 2.15-66.27, \mathrm{P}=0.005)$. Similarly, combined analysis of another two trials $[20,23]$ revealed that the immune response positivity rate in the sarcoidosis group was significantly higher than that in the subgroup of controls with an unknown PPD status ( $\mathrm{OR}=41.97,95 \%$ CI: 5.24-336.15, P = 0.0004) (Fig 5). Heterogeneity test revealed that there was not substantial heterogeneity in either of these two subgroups, as assessed using the $\mathrm{I}^{2}$ statistic (subgroup of PPD- controls $(\mathrm{Q}(\mathrm{d} . \mathrm{f} .=1)=0.28$, $\left.\mathrm{P}=0.60, \mathrm{I}^{2}=0 \%\right)$; subgroup of controls with unknown PPD status $(\mathrm{Q}(\mathrm{d} . \mathrm{f} .=1)=0.14$, $\left.\mathrm{P}=0.71, \mathrm{I}^{2}=0 \%\right)$ (Fig 5). The heterogeneity between these two subgroups was also not substantial $\left(\mathrm{Q}(\mathrm{d} . \mathrm{f} .=1)=0.84, \mathrm{P}=0.36, \mathrm{I}^{2}=0 \%\right)$. Publication bias was not evident, as estimated by generation of a funnel plot for the studies on the T-cell immune response to M. tuberculosis specific antigens (Fig 4c).

\section{Discussion}

Meta-analysis was the main method used in this research paper. It is more accurate and reliable than regression analysis or original papers. 
The relationship between sarcoidosis and $M$. tuberculosis has been a highly contested and challenging issue. Further analysis is important considering their opposite therapeutic effects In the current meta-analysis, a greater positivity incidence of the immune response to M. tuberculosis specific antigens was observed in sarcoidosis group compared to the control group of subjects with PPD- or unknown PPD status for both the T-cell immune response and humoral response, indicating that these antigens induce T-cell-specific responses in the blood or BALF of sarcoidosis patients. However, it is important to note that sarcoidosis patients may be more likely to have positive TST results (i.e. to be more sensitive to LTBI) than healthy individuals due to unknown host factors with no known etiologic link between sarcoidosis and M. tuberculosis. Notably, the positivity incidence of T-cell immune response to M. tuberculosis specific antigens in sarcoidosis group was found to be significantly lower in the sarcoidosis patients than in the PPD+ (LTBI) controls, although the incidences did not differ among the four primary trials. Further, it was hypothesized that $M$. tuberculosis specific antigens response would not be observed in all sarcoidosis patients, and that the specific mycobacterial antigen may represent just one of the most important factors for the pathogenesis of sarcoidosis.

In the T-cell immune response, no significant heterogeneity was observed in either the subgroup of PPD- controls or in the subgroup of controls with an unknown PPD status. Similarly, no significant heterogeneity was detected in the group of PPD+ (LTBI) controls. Similarly, no significant heterogeneity was detected in the group of PPD+ (LTBI) controls. Further, in the humoral response, no significant heterogeneity was observed in either the subgroup of PPDcontrols or the subgroup of controls with an unknown PPD status. However, significant heterogeneity was detected between the two subgroups with PPD- or unknown PPD status on the $\mathrm{T}$-cell immune response, indicating that PPD status strongly influenced experimental outcome.

A number of original studies have examined mycobacteria in samples of sarcoidosis patients [7, 25-32], with different results. In addition, a meta-analysis [9] on the molecular evidence of the role of mycobacteria in sarcoidosis conducted in 2007 demonstrated mycobacterial presence in sarcoidosis lesions, suggesting the presence of an association between mycobacteria and sarcoidosis in some cases. However, it is important to emphasize that the mere presence of mycobacterial DNA does not indicate the existence of a cause-and-effect relationship. In 1998, evidence that the granulomatous response is driven by tissue antigens in sarcoidosis was provided by analysis of TCR expression [33]. Many recent studies have reported that $M$. tuberculosis antigens are involved in the pathogenesis of sarcoidosis [10, 34]. Among our included studies, one study conducted by Song reported the detection of several M. tuberculosis antigens including catalase-peroxidase antigen (mKatG) and Mtb $16 \mathrm{~S}$ rRNA in nearly $40 \%$ of their sarcoidosis subjects as detected by in situ hybridization [22]. Further, some studies have suggested that some antigens of M. tuberculosis (and not the whole mycobacteria) are involved in the pathogenesis of sarcoidosis $[5,16]$. These data suggest the presence of mycobacterial proteins preferentially in sarcoidosis patients. Indeed, some factors do favor the involvement of mycobacteria in sarcoidosis, including the following: (1) histopathological appearances of the granulomas; (2) an immune response to M. tuberculosis antigens in sarcoidosis patients; (3) development of mycobacterial disease either coincidentally, before or after development of sarcoidosis [35-37]; (4) the detection of mycobacteria from samples of patients with sarcoidosis by PCR $[26,27]$; and (5) acid-fast cell wall-deficient forms (CWDF) of bacteria can be grown from the blood of patients with sarcoidosis $[38,39]$. It is important to note that mycobacterial (TB or NTM) antigens and not the active mycobacteria may play roles in the pathogenesis of sarcoidosis. One hypothesis is that an impediment in the removal of poorly degraded antigenic material may contribute to the pathogenesis of sarcoidosis [22, 40]. However, it is important to note that mycobacterial antigens alone cannot explain the pathogenesis of this disease in all 
subjects, as no immune response to M. tuberculosis antigens or molecular evidence was observed in some sarcoidosis subjects. Therefore, we assumed that other factors besides mycobacterial antigens contribute to the pathogenesis of sarcoidosis.

There are several limitations to this study. First, although data were collected from more than 700 patients, the number of incorporated studies was still small, and therefore these results may have been affected by publication bias. Second, the definite sensitivity of the immunological data is unknown, as they were not described or analyzed in detail in this study. Third, some variations may also have occurred due to differences in local environmental exposure and the fact that some sarcoidosis patients may have a disorder initiated by organisms or triggers other than mycobacteria. Therefore, additional studies would be useful to provide more concrete data to investigate the accuracy of this conclusion are needed.

In conclusion, the results of this meta-analysis have demonstrated an association between mycobacteria (especially M.tuberculosis) and sarcoidosis. Mycobacteria was not observed in all cases of sarcoidosis, however, these bacteria indeed represent an important factor involved in the pathogenesis of this disease. It has been hypothesized that only some specific, poorly degraded mycobacterial pathogenic antigens contribute to immune-mediated granulomatous inflammation rather than the whole active mycobacteria and that they elicit a type IV immune response.

\section{Supporting Information}

S1 Table. PRISMA checklist of this meta-analysis.

(DOC)

\section{Author Contributions}

Conceived and designed the experiments: CLF ZJX.

Performed the experiments: CLF HH.

Analyzed the data: CLF.

Contributed reagents/materials/analysis tools: CLF.

Wrote the paper: CLF ZJX.

\section{References}

1. Agarwal A, Prasad R, Jain A. Effect of green tea extract (catechins) in reducing oxidative stress seen in patients of pulmonary tuberculosis on DOTS Cat I regimen. PHYTOMEDICINE. [Journal Article; Randomized Controlled Trial; Research Support, Non-U.S. Gov't]. 2010 2010-01-01; 17(1):23-7. doi: 10. 1016/j.phymed.2009.10.019 PMID: 19910173

2. Brownell I, Ramirez-Valle F, Sanchez M, Prystowsky S. Evidence for mycobacteria in sarcoidosis. Am J Respir Cell Mol Biol. [Journal Article; Review]. 2011 2011-11-01; 45(5):899-905. doi: 10.1165/rcmb. 2010-0433TR PMID: 21659662

3. Dubaniewicz A. Mycobacterium tuberculosis heat shock proteins and autoimmunity in sarcoidosis. AUTOIMMUN REV. [Journal Article; Research Support, Non-U.S. Gov't; Review]. 2010 2010-04-01; 9 (6):419-24. doi: 10.1016/j.autrev.2009.11.015 PMID: 19931650

4. Gupta D, Agarwal R, Aggarwal AN, Jindal SK. Sarcoidosis and tuberculosis: the same disease with different manifestations or similar manifestations of different disorders. CURR OPIN PULM MED. [Journal Article; Review]. 2012 2012-09-01; 18(5):506-16. PMID: 22759770

5. Oswald-Richter KA, Beachboard DC, Zhan X, Gaskill CF, Abraham S, Jenkins C, et al. Multiple mycobacterial antigens are targets of the adaptive immune response in pulmonary sarcoidosis. RESP RES. 2010 2010-01-01; 11. 
6. SCADDING JG. Mycobacterium tuberculosis in the aetiology of sarcoidosis. Br Med J. [Journal Article]. 1960 1960-12-03; 2(5213):1617-23. PMID: 13747016

7. Eishi Y, Suga M, Ishige I, Kobayashi D, Yamada T, Takemura T, et al. Quantitative analysis of mycobacterial and propionibacterial DNA in lymph nodes of Japanese and European patients with sarcoidosis. J CLIN MICROBIOL. [Journal Article; Research Support, Non-U.S. Gov't]. 2002 2002-01-01; 40 (1):198-204. PMID: 11773116

8. Li N, Bajoghli A, Kubba A, Bhawan J. Identification of mycobacterial DNA in cutaneous lesions of sarcoidosis. J CUTAN PATHOL. 1999 1999-01-01; 26(6):271-8. PMID: 10472755

9. Gupta D, Agarwal R, Aggarwal AN, Jindal SK. Molecular evidence for the role of mycobacteria in sarcoidosis: a meta-analysis. EUR RESPIR J. [Journal Article; Meta-Analysis]. 2007 2007-09-01; 30 (3):508-16. PMID: 17537780

10. Ang SC, Moscovic EA. Cross-reactive and species specific Mycobacterium tuberculosis antigens in the immunoprofile of Schaumann bodies: A major clue to the etiology of sarcoidosis. HISTOL HISTOPATHOL. 1996 1996-01-01; 11(1):125-34. PMID: 8720456

11. Inui N, Suda T, Chida K. Use of the QuantiFERON-TB Gold test in Japanese patients with sarcoidosis. RESP MED. 2008 2008-01-01; 102(2):313-5.

12. Hofland RW, Thijsen SFT, Bouwman J, Van Der Wel M, Bossink AWJ. Sarcoidosis and purified protein derivative reactivity. SARCOIDOSIS VASC DIF. 2014 2014-01-01; 31(2):142-8.

13. Oswald-Richter KA, Beachboard DC, Seeley EH, Abraham S, Shepherd BE, Jenkins CA, et al. Dual analysis for mycobacteria and propionibacteria in sarcoidosis BAL. J CLIN IMMUNOL. 2012 2012-0101; 32(5):1129-40. doi: 10.1007/s10875-012-9700-5 PMID: 22552860

14. Gupta D, Kumar S, Aggarwal AN, Verma I, Agarwal R. Interferon gamma release assay (QuantiFERON-TB gold in tube) in patients of sarcoidosis from a population with high prevalence of tuberculosis infection. SARCOIDOSIS VASC DIF. 2011 2011-01-01; 28(2):95-101.

15. Horster R, Kirsten D, Gaede KI, Jafari C, Strassburg A, Greinert U, et al. Antimycobacterial immune responses in patients with pulmonary sarcoidosis. CLIN RESPIR J. [Journal Article]. 2009 2009-10-01; 3(4):229-38. doi: 10.1111/j.1752-699X.2009.00136.x PMID: 20298409

16. Oswald-Richter KA, Culver DA, Hawkins C, Hajizadeh R, Abraham S, Shepherd BE, et al. Cellular responses to mycobacterial antigens are present in bronchoalveolar lavage fluid used in the diagnosis of sarcoidosis. INFECT IMMUN. [Journal Article; Research Support, N.I.H., Extramural]. 2009 2009-0901; 77(9):3740-8. doi: 10.1128/IAl.00142-09 PMID: 19596780

17. Hajizadeh R, Sato H, Carlisle J, Nadaf MT, Evans W, Shepherd BE, et al. Mycobacterium tuberculosis antigen 85A induces Th-1 immune responses in systemic sarcoidosis. J CLIN IMMUNOL. 2007 200701-01; 27(4):445-54. PMID: 17357846

18. Drake WP, Dhason MS, Nadaf M, Shepherd BE, Vadivelu S, Hajizadeh R, et al. Cellular recognition of Mycobacterium tuberculosis ESAT-6 and KatG peptides in systemic sarcoidosis. INFECT IMMUN. 2007 2007-01-01; 75(1):527-30. PMID: 17088357

19. Carlisle J, Evans W, Hajizadeh R, Nadaf M, Shepherd B, Ott RD, et al. Multiple Mycobacterium antigens induce interferon-gamma production from sarcoidosis peripheral blood mononuclear cells. CLIN EXP IMMUNOL. [Journal Article; Research Support, N.I.H., Extramural]. 2007 2007-12-01; 150 (3):460-8. PMID: 17924974

20. Agarwal R, Gupta D, Srinivas R, Verma I, Aggarwal AN, Laal S. Analysis of humoral responses to proteins encoded by region of difference 1 of Mycobacterium tuberculosis in sarcoidosis in a high tubercuIosis prevalence country. INDIAN J MED RES. [Letter; Research Support, N.I.H., Extramural]. 2012 2012-06-01; 135(6):920-3. PMID: 22825614

21. Dubaniewicz A, Dubaniewicz-Wybieralska M, Sternau A, Zwolska Z, Izycka-Swieszewska E, Augustynowicz-Kopec E, et al. Mycobacterium tuberculosis complex and mycobacterial heat shock proteins in lymph node tissue from patients with pulmonary sarcoidosis. J CLIN MICROBIOL. [Journal Article; Research Support, Non-U.S. Gov't]. 2006 2006-09-01; 44(9):3448-51. PMID: 16954298

22. Song Z, Marzilli L, Greenlee BM, Chen ES, Silver RF, Askin FB, et al. Mycobacterial catalase-peroxidase is a tissue antigen and target of the adaptive immune response in systemic sarcoidosis. J EXP MED. 2005 2005-01-01; 201(5):755-67. PMID: 15753209

23. El-Zaatari FA, Naser SA, Markesich DC, Kalter DC, Engstand L, Graham DY. Identification of Mycobacterium avium complex in sarcoidosis. J CLIN MICROBIOL. [Journal Article; Research Support, Non-U. S. Gov't; Research Support, U.S. Gov't, Non-P.H.S.]. 1996 1996-09-01; 34(9):2240-5. PMID: 8862592

24. Ahmadzai H, Cameron B, Chui JJ, Lloyd A, Wakefield D, Thomas PS. Peripheral blood responses to specific antigens and CD28 in sarcoidosis. Respir Med. [Journal Article; Multicenter Study]. $20122012-$ 05-01; 106(5):701-9. doi: 10.1016/j.rmed.2012.01.012 PMID: 22349068 
25. Mootha VK, Agarwal R, Aggarwal AN, Gupta D, Ahmed J, Verma I, et al. The Sarcoid-Tuberculosis link: Evidence from a high TB prevalence country. J INFECTION. 2010 2010-01-01; 60(6):501-3.

26. Ding XL, Cai L, Zhang JZ. Detection and identification of mycobacterial gene in skin lesions and lymph nodes in patients with sarcoidosis. Acta Academiae Medicinae Sinicae. 2009 2009-01-01; 31(1):20-3. PMID: 19317051

27. Zhou Y, Li HP, Li QH, Zheng H, Zhang RX, Chen G, et al. Differentiation of sarcoidosis from tuberculosis using real-time PCR assay for the detection and quantification of Mycobacterium tuberculosis. SARCOIDOSIS VASC DIF. 2008 2008-01-01; 25(2):93-9.

28. Li QH, Zhao L, Li HP, Shen YP, Zhang RX, Zheng H, et al. [Application of quantitative polymerase chain reaction in the differentiation of sarcoidosis and proliferative tuberculosis]. Zhonghua Jie $\mathrm{He} \mathrm{He}$ Hu Xi Za Zhi. [English Abstract; Evaluation Studies; Journal Article; Research Support, Non-U.S. Gov't]. 2007 2007-09-01; 30(9):686-90. PMID: 18070554

29. Yasuhara T, Tada R, Nakano Y, Tei M, Mochida C, Kamei M, et al. The presence of Propionibacterium spp. in the vitreous fluid of uveitis patients with sarcoidosis. Acta Ophthalmologica Scandinavica. 2005 2005-01-01; 83(3):364-9. PMID: 15948792

30. Milman N, Lisby G, Friis S, Kemp L. Prolonged culture for mycobacteria in mediastinal lymph nodes from patients with pulmonary sarcoidosis. A negative study. Sarcoidosis Vasc Diffuse Lung Dis. [Journal Article]. 2004 2004-03-01; 21(1):25-8. PMID: 15127971

31. Hofman V, Selva E, Landraud L, Sicard D, Venissac N, Castillo L, et al. [Value of PCR amplification from formalin-fixed paraffin-embedded tissues in the diagnosis of Mycobacterium tuberculosis infection]. ANN PATHOL. [English Abstract; Journal Article; Research Support, Non-U.S. Gov't]. 2003 200306-01; 23(3):206-15. PMID: 12909824

32. Drake WP, Pei Z, Pride DT, Collins RD, Cover TL, Blaser MJ. Molecular analysis of sarcoidosis tissues for Mycobacterium species DNA. EMERG INFECT DIS. 2002 2002-01-01; 8(11):1334-41. PMID: 12453366

33. Moller DR. Involvement of T cells and alterations in T cell receptors in sarcoidosis. Semin Respir Infect. [Journal Article; Research Support, U.S. Gov't, P.H.S.; Review]. 1998 1998-09-01; 13(3):174-83. PMID: 9764948

34. Litvinov VI, Chernousova LN, Safonova SG, Kalinina OA, Kulikovskaia NV, Kapina MA, et al. [Interaction of Mycobacteria isolated from sarcoidosis patients with antituberculosis antibodies]. Probl Tuberk. [English Abstract; Journal Article]. 1997 1997-01-19(5: ):36-8. PMID: 9454251

35. Piotrowski WJ, Gorski P, Duda-Szymanska J, Kwiatkowska S. Mycobacterium tuberculosis as a sarcoid factor? A case report of family sarcoidosis. Am J Case Rep. [Journal Article]. 2014 2014-01-20; 15:216-20. doi: 10.12659/AJCR.890014 PMID: 24847413

36. van Enschot JW, van Balkom RH. Sarcoidosis following Mycobacterium tuberculosis infection: Coincidence or consequence. Respir Med Case Rep. [Journal Article]. 2013 2013-01-20; 9:11-4. doi: 10. 1016/j.rmcr.2013.03.006 PMID: 26029621

37. Luk A, Lee A, Ahn E, Soor GS, Ross HJ, Butany J. Cardiac sarcoidosis: recurrent disease in a heart transplant patient following pulmonary tuberculosis infection. CAN J CARDIOL. [Case Reports; Journal Article]. 2010 2010-08-01; 26(7):e273-5. PMID: 20847976

38. Brown ST, Brett I, Almenoff PL, Lesser M, Terrin M, Teirstein AS. Recovery of cell wall-deficient organisms from blood does not distinguish between patients with sarcoidosis and control subjects. CHEST. 2003 2003-01-01; 123(2):413-7. PMID: 12576359

39. Almenoff PL, Johnson A, Lesser M, Mattman LH. Growth of acid fast $L$ forms from the blood of patients with sarcoidosis. THORAX. 1996 1996-01-01; 51(5):530-3. PMID: 8711683

40. Saunders BM, Cooper AM. Restraining mycobacteria: role of granulomas in mycobacterial infections. IMMUNOL CELL BIOL. [Journal Article; Research Support, Non-U.S. Gov't]. 2000 2000-08-01; 78 (4):334-41. PMID: 10947857 